\title{
Modelling skin temperature of a human exercising in an outdoor environment
}

\author{
J. K. Vanos ${ }^{1}$, J. S. Warland ${ }^{1}$, N. A. Kenny ${ }^{2} \&$ T. J. Gillespie ${ }^{1}$ \\ ${ }^{1}$ School of Environmental Sciences, University of Guelph, Canada \\ ${ }^{2}$ Teaching Support Services, University of Guelph, Canada
}

\begin{abstract}
Skin temperature monitoring is an important component when estimating thermoregulatory responses due to heat exchange at the skin surface. The aim of this study is to improve the accuracy of mean skin temperature $\left(\bar{T}_{s k}\right)$ predictions in human thermal comfort models, specifically the COMFA (COMfort FormulA) outdoor model, in order to reduce errors in energy budget estimates associated with $\bar{T}_{s k}$. Field tests were conducted on 12 subjects performing 30 minutes of steadystate physical activity (running or cycling) on two separate occasions. The predicted thermal sensations (PTS) from the COMFA budget model using both actual (measured) and predicted (with model) $\bar{T}_{s k}$ were compared at 5-minute intervals.

Results indicate that the model over-predicted $\bar{T}_{s k}$ throughout the exercise period. The root mean square error (RMSE) of $\bar{T}_{s k}$ of subjects running was larger than cycling, and increased throughout the 30 minute exercise session; hence, the model was less able to accurately predict $\bar{T}_{s k}$ as metabolic activity increased. The Spearman's correlation coefficients $\left(\mathrm{r}_{s}\right)$ for actual thermal sensation (ATS) with both actual and predicted $\bar{T}_{s k}$ thermal sensation scores were low, $\left(\mathrm{r}_{s}=0.315\right.$ and 0.285 , respectively). However, ATS votes correlated more strongly with predicted thermal sensation (PTS) scores in running tests. Added psychological and physiological variables when exercising outdoors make it inherently difficult to apply current thermal comfort (TC) models to exercising subjects. There is need for further studies regarding prediction of overall TC while exercising outdoors for use in urban design and planning, as well as adapting models to specific types of exercise.
\end{abstract}

Keywords: outdoor human comfort, bioclimatic design, COMFA model, physical activity, skin temperature. 


\section{Introduction}

A thermally comfortable outdoor space influences the type and amount of physical activity a person performs, and creation of comfortable spaces is needed to meet the demand of outdoor recreational users [1]. The future growth of cities will undoubtedly cause further problems associated with cardiovascular and respiratory problems, as well as death and illness from heat and air pollutants during the warmest times of the year. Detrimental impacts can be further expanded to athletic performance, work and behaviour [2]. Many studies have specifically noted that urban dwellers show overall decreased health and functionality in everyday life [3], and also higher heat related mortality [4, 5]. Through the use of accurate TC models applied to bioclimatic urban design, improvement of outdoor spaces more conducive to recreational activities can be achieved.

TC models are used to predict how a human 'feels' physiologically in their given environment. Most well-validated heat budget models, such as that by Fanger [6] or Gagge [7], have been developed from indoor laboratory studies (steady-state) that are non-complex $[8,9]$. These are not always appropriate for measuring outdoor heat stress and TC due to their inability to accurately represent the ambient environment [2], or account for differing physiological responses and psychological perceptions of indoor and outdoor environments [9].

The main function of local $T_{s k}$ and $\bar{T}_{s k}$ is to aid in regulation of bloodflow [8], and hence heat distribution and loss throughout the body. The ability to accurately measure $\bar{T}_{s k}$ of a human is difficult due to temperature variations of the skin, which become more complex during exercise and sweating [10]. Thermal comfort is greatly determined by $\bar{T}_{s k}$ [11]. Actual thermal sensation (ATS) is the perception of heat or cold (what one feels). Yao et al [11] found that the conscious mind reaches conclusions about ATS directly from the temperature of the skin. One can sense a change in ATS through thermoreceptors located in the skin, with a high rate of temperature change $(\Delta T)$ causing a greater effect on ATS.

The purpose of this study was to use the COMFA outdoor model to predict $\bar{T}_{s k}$ values $\left(\bar{T}_{P s k}\right)$ and statistically compare these to actual $\bar{T}_{s k}\left(T_{A s k}\right)$ of subjects performing moderate-vigorous physical activity outdoors. This will determine the reliability of model predictions under varying weather conditions and metabolic rates.

\section{Methods}

Methods pertaining to data acquisition of meteorological and physiological data will be outlined in the following section. This data was incorporated into the COMFA model and compared with subjective responses provided by subjects during their exercise sessions in an outdoor environment.

\subsection{Field surveys, participants and physiological data collection}

A total of 12 physically active and healthy subjects (six female, six male) between the ages of 19 and 23 participated in the study. All subjects were healthy and active 
in their daily lives, accustomed to exercising at moderate to intense training levels approximately 4-5 times per week. The average age, weight and height of the female participants was 21.5 years, $170.9 \mathrm{~cm}$, and $62.1 \mathrm{~kg}$, while the average for males was 21.8 years, $186.5 \mathrm{~cm}$, and $81.8 \mathrm{~kg}$, respectively. Subjects abstained from strenuous activity, caffeine and use of sunscreen or lotion on the day of the test, and did not eat within 1-hour prior to the sampling period. During the 30 minute study period, subjects were not permitted to drink any fluid.

Each participant completed either two cycling or two running exercise sessions of 30 minutes in length. Cycling tests were completed on the dates of July 16 and 30, 2009, while jogging tests were completed on September 16 and 30, 2009, between the hours of $1100 \mathrm{hr}$ and $1900 \mathrm{hr}$. All tests were completed at the University of Guelph campus $\left(43.33^{\circ} \mathrm{N}, 80.2^{\circ} \mathrm{W}, 377 \mathrm{~m}\right.$ above sea level) on large, uniform grass multipurpose fields, away from any shading from buildings or trees.

The test subjects wore comfortable clothing appropriate for the environmental conditions and exercise activity. For tests on the first three days (warm, fair weather), all subjects wore white cotton T-shirts with regular athletic shorts and shoes. On the fourth day, subjects were allowed to wear clothing suitable for the cooler weather. Clothing ensembles were assigned in accordance with ISODIS 9920 [12] from which static clothing resistances $\left(r_{c o}\right)$ and static clothing vapour resistances $\left(r_{c v o}\right)\left(\mathrm{s} \mathrm{m}^{-1}\right)$ were found. If the exact clothing ensemble was not found in the listed ensembles, the total insulation value $\left(I_{c l}\right)$ (clo) was found using eqn. (1) [12], where a clo is an arbitrary unit of clothing insulation (1 clo = $\left.186.6 \mathrm{~s} \mathrm{~m}^{-1}=0.1555 \mathrm{~m}^{2}{ }^{o} \mathrm{C}^{-1} W^{-1}\right)$.

$$
I_{c l}=0.161+0.836 \sum I_{c l u}
$$

where $I_{c l u}$ is the effective thermal insulation of individual garments making up the ensemble, and $I_{c l}$ values were converted to $r_{c o}$ using $=I_{c l} \times 186.6 \mathrm{sm}^{-1}$.

Two cycling ergonometers (Monark, Ergomedic 874E) were used for the cycling tests, positioned in close proximity $(10-20 \mathrm{~m})$ to the portable weather station (See Section 2.3). The ergonometers measured power output (W), speed $\left(\mathrm{m} \mathrm{s}^{-1}\right)$, and revolutions per minute (RPM) of the cyclers. Metabolic activity rates $\left(M_{a c t}\right)$ $\left(\mathrm{W} \mathrm{m}{ }^{-2}\right.$ ) were estimated from the power output of the cyclists and the speed of the runners using the activity codes and MET intensities listed in Ainsworth et al [13], where $1 \mathrm{MET}=58.15 \mathrm{~W} \mathrm{~m}^{-2}$ [14].

\subsection{Physiological and psychological data acquisition}

Using a structured three-question survey, participants were asked to rate their ATS (7-point scale) [6], rate of perceived exertion (RPE) [15] and preferred change (PC) in sensation every 5 minutes during the study. Subjects were asked to focus on overall feelings of exertion and sensation, rather than specific areas of the body. The subjects provided their radial neck pulse count over a 10 second period every 5 minutes. 
Subjects exercised at a moderate intensity of 60-69\% max HR. This is equivalent to an RPE of 12-13 ("somewhat hard"), that which is prescribed by the American College of Sports Medicine, which recommends 30 minutes of moderate exercise 4-7 times per week [16]. Each subject's HR range was based on their age and resting heart rate, using the Karvonen method to find target heart rate [17] at the set intensity.

Each participant had fast response thermocouples (SA1-T Omega Engineering, Stamford, CT) attached to their left calf, right thigh, right upper arm and left chest [10]. $\bar{T}_{s k}$ was calculated using the Ramanathan 4-point weighting method (eqn. (2)).

$$
\bar{T}_{s k}=0.3 T_{\text {chest }}+0.3 T_{\text {arm }}+0.2 T_{\text {leg }}+0.2 T_{\text {thigh }}
$$

Skin temperature readings were recorded using a 21X datalogger (Campbell Scientific Instruments, Logan, UT) for the cycling tests and a $164 \times 76 \times 32 \mathrm{~mm}$ portable datalogger (HH147, Omega Engineering, Stamford, CT) secured on a waist belt during the running tests. All skin temperature data were collected at 10 -second intervals and calculated as 5 minute averages over the sampling period.

\subsection{Meteorological data acquisition}

Absorbed radiation was measured using a Cylindrical Radiation Thermometer (CRT) as modified by Kenny et al [16] mounted at $90^{\circ}$ to the ground surface, painted to have an albedo of 0.37 and an emissivity of 0.95 , representing the radiative properties of a human. It was ensured that no shadows were cast on either of the radiation instruments. Wind velocity $\left(v_{w}\right)$ was measured using a cup anemometer (03102 R.M. Young Wind Sentry Anemometer, Campbell Scientific, Logan, UT). $R H$ and $T_{a}$ were measured using an $\mathrm{HC}-\mathrm{S} 3$ temperature and relative humidity probe (Campbell Scientific, Logan, UT). All instruments were mounted on a tripod (Hoskin Scientific, Burlington, ON) 1.5-2.0 m above the ground surface. All meteorological data were collected with a CR21X datalogger (Campbell Scientific Instruments, Logan, UT) at 10-second intervals and calculated as 5 minute averages.

\subsection{Modeling thermal comfort using the COMFA model}

The energy budget for each participant was calculated every 5 minutes during the exercise session based on the COMFA outdoor thermal comfort model $[1,16]$. The COMFA model requires the following inputs: $T_{a}\left({ }^{\circ} \mathrm{C}\right), R H(\%), v_{w}\left(\mathrm{~m} \mathrm{~s}^{-1}\right)$, clothing insulation $\left(\mathrm{s} \mathrm{m}^{-1}\right)$, clothing vapour insulation $\left(\mathrm{s} \mathrm{m}^{-1}\right)$, metabolic activity $\left(M_{a c t}\right)\left(\mathrm{W} \mathrm{m}^{-2}\right)$ and total absorbed radiation $\left(\mathrm{W} \mathrm{m}^{-2}\right)$. Integral changes to equations involving clothing resistance $\left(r_{c}\right)$, clothing vapour resistance $\left(r_{c v o}\right)$, tissue resistance $\left(r_{t}\right)$ and $\bar{T}_{s k}$ (eqn. (3)), as proposed by Kenny et al [18] for exercising individuals, were incorporated in the current study. 
Table 1: Summary of meteorological conditions recorded on-site expressed as a mean over each test period

\begin{tabular}{lccccc}
\hline Date & Time $(\mathrm{hr})$ & $\mathrm{T}_{a}\left({ }^{\circ} \mathrm{C}\right)$ & $e(\mathrm{kPa})$ & $v_{w}\left(\mathrm{~ms}^{-1}\right)$ & $T_{R T}\left({ }^{\circ} \mathrm{C}\right)$ \\
\hline $16 / 07 / 09$ & $1325-1650$ & 24.4 & 1.4 & 2.8 & 26.7 \\
$30 / 07 / 09$ & $1320-1640$ & 24.3 & 1.4 & 2.7 & 26.3 \\
$16 / 09 / 09$ & $1245-1535$ & 16.7 & 1.0 & 1.9 & 19.7 \\
$30 / 09 / 09$ & $1230-1530$ & 8.9 & 0.7 & 2.5 & 10.4 \\
\hline
\end{tabular}

${ }^{a} T_{a}$ (air temperature), $e$ (ambient vapour pressure, $v_{w}$ (wind speed), $T_{R T}$ (CRT temperature), expressed as a mean over the test periods.

$$
\bar{T}_{s k}=\left(\frac{T_{c}-T_{a}}{r_{t}+r_{c}+r_{a}}\right)\left(r_{a}+r_{c}\right)+T_{a}
$$

$T_{c}$ is core temperature, and $r_{t}, r_{c}$ and $r_{a}$ are tissue, clothing and aerodynamic resistances, respectively ( $\mathrm{s} \mathrm{m}^{-1}$ ). Kenny et al [18] used eqn. (3), which makes use of Ohm's Law Analogy and $T_{a}$. This was found to produce values consistent with research completed by Mairiaux et al [19], and Sparks et al [20].

\section{Results and discussion}

\subsection{Overview of microclimatic conditions}

A total of 12 field tests were conducted on four different days under varying temperature and cloud conditions, and on non-rainy days. A summary of the meteorological conditions on each of the field testing days is shown in Table 1.

\subsection{Overview of participant physiological data}

The mean heart rate (HR) of the cyclists was 134 BPM (range = 54-176 BPM), while that of the runners was 139 BPM (range $=54-180$ BPM). During the testing periods, $57 \%$ of the cyclists reached their target HR (THR) of $60 \%$ intensity within 10 minutes of starting exercise, while $29 \%$ of the subjects surpassed their upper THR limit of $69 \%$ intensity by $3-19 \%$. The rate of increase in the HR was greater for runners than for cyclists with $64 \%$ reaching their $60 \%$ THR within 10 minutes, and $43 \%$ surpassing their upper THR by $2-18 \%$.

The mean speed of the cyclists was $9.3 \mathrm{~m} \mathrm{~s}^{-1}$ (range $\left.=6.7-18.0 \mathrm{~m} \mathrm{~s}^{-1}\right)$. The mean observed speed of the runners was $3.3 \mathrm{~m} \mathrm{~s}^{-1}$ (range $\left.=2.5-3.8 \mathrm{~m} \mathrm{~s}^{-1}\right) . M_{a c t}$ varied between individual subjects and exercise type. The mean $M_{a c t}$ for combined cycling and running tests was $476 \mathrm{~W} \mathrm{~m}^{-2}$ (range $=87 \mathrm{~W} \mathrm{~m}^{-2}$ at rest to 


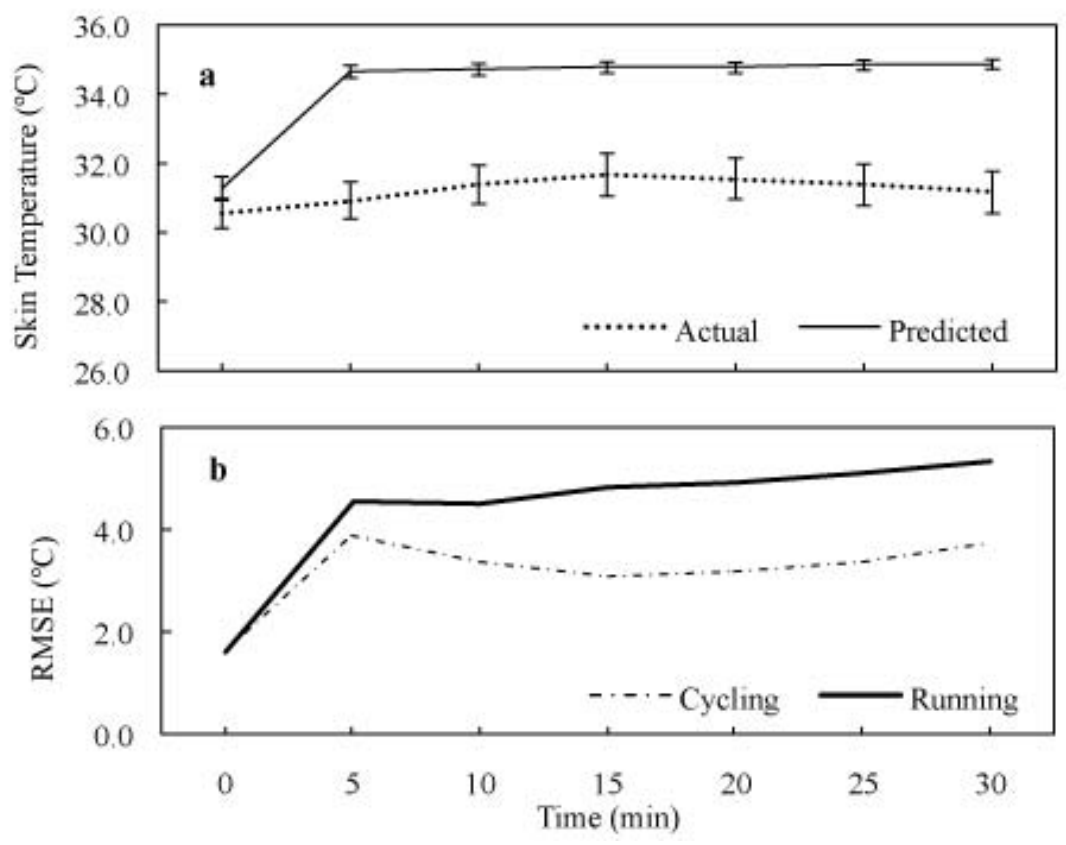

Figure 1: a) Average actual $\left(\bar{T}_{A s k}\right)$ and predicted $\left(\bar{T}_{P s k}\right)$ mean skin temperature; b) RMSE variations during 30 minute exercise session. Error bars \pm 1 standard error.

$\left.775 \mathrm{~W} \mathrm{~m}^{-2}\right)$. Mean $M_{\text {act }}$ for cycling was $332 \mathrm{~W} \mathrm{~m}^{-2}\left(\right.$ range $\left.=87-510 \mathrm{~W} \mathrm{~m}^{-2}\right)$, while for running was 621 (range $=87-835 \mathrm{~W} \mathrm{~m}^{-2}$ ).

\subsection{Comparison of actual and predicted skin temperatures}

Statistical evaluation of the difference between the actual and predicted $\bar{T}_{s k}$ at each time interval from 0-30 minutes was conducted using the Root Mean Square Error (RMSE). The 5 minute variation of the RMSE $\left({ }^{\circ} \mathrm{C}\right)$ for all time periods during the experiments $(n=168)$ was 3.8 , with separate cycling and running tests $(n=84)$ have a RMSE of 3.2 and 4.4, respectively. Figure 1 displays the change of $\bar{T}_{A s k}$ and $\bar{T}_{P s k}$ with time, as well as RMSE over time, where $\bar{T}_{A s k}$ is a weighted average (eqn. (3)), and $\bar{T}_{P s k}$ is estimated using the COMFA model.

This shows that the model is over predicting $\bar{T}_{s k}$ throughout the exercise period, excluding $\mathrm{t}=0$, with running showing a larger RMSE and over prediction than cycling. Residuals between actual and predicted $\bar{T}_{s k}$ were significantly different between exercises $(\mathrm{p}<0.05)$; thus, it is more difficult to predict $\bar{T}_{s k}$ of a human while running. This supports recommendations made by Brotherhood [2] for sportspecific heat stress algorithms. The incorporation of metabolic heat production, 


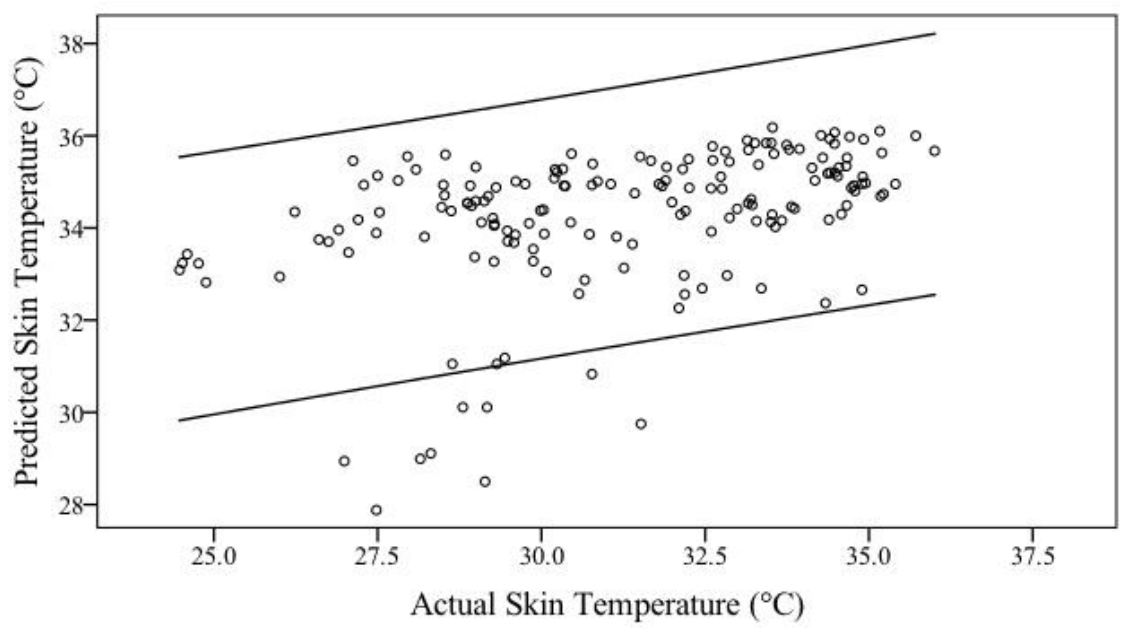

Figure 2: Scatter plot displaying relationship between actual mean skin temperature $\left(\bar{T}_{A s k}\right)$ and predicted $\left(\bar{T}_{P s k}\right)\left(\mathrm{R}^{2}=0.173\right)$ with $95 \%$ confidence intervals.

plus effects of body movement and clothing, are not included in heat stress indices, such as the Wet Bulb Globe Thermometer, and therefore cannot accurately predict human comfort while exercising [2]. The COMFA model incorporates all mentioned components; however, the cyclists were stationary, and thus an activity speed $\left(v_{a}\right)$ was not present, whereas running did make use of $v_{a}$ in the model. The lower vapour pressure during the running tests on days 3 and 4, plus the addition of body movement to increase vapour loss, resulted in significantly higher levels of evaporation during the latter two days $(\mathrm{p}<0.05)$.

Figure 2 displays the correlation between $\bar{T}_{A s k}$ and $\bar{T}_{P s k}$, with an $\mathrm{R}^{2}$ of 0.173 . Of the nine data points outside the $95 \%$ confidence interval, all were at $t=0$ minutes prior to the start of exercise on test days 3 and 4. The RMSE for running shows an increasing trend throughout, which follows the trend of metabolic activity $\left(M_{a c t}\right)$ and HR, which were greater for running than cycling. Runners surpassed their THR $43 \%$ of the time, vs. $29 \%$ for cyclists, and therefore were exercising at a higher average intensity than that of the cyclists. These results show that the model tends to over predict $\bar{T}_{s k}$ to a larger extent with higher $M_{a c t}$, and thus the inherent difficulty in using TC for exercising individuals. Additional variables, such as fitness, physiological strain, metabolism, core temperature and sweat rate may need to be considered in such situations.

\subsubsection{Comparing actual and predicted thermal sensation scores}

Through the use of the updated budget values by Kenny et al [18], the ATS given by the subjects and PTS found by the full COMFA model were categorized based 

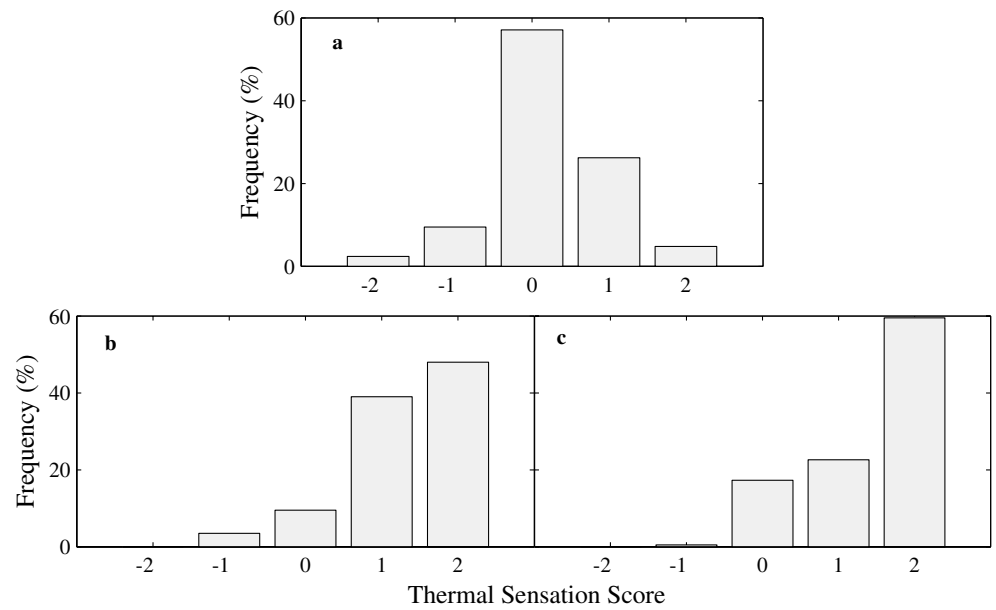

Figure 3: Histograms displaying frequency distributions of a) actual thermal sensation (ATS) given by subjects; b) predicted thermal sensation (PTS) using predicted mean skin temperature $\left.\left(\bar{T}_{P s k}\right) ; \mathrm{c}\right)$ PTS using actual mean skin temperature $\left(\bar{T}_{\text {Ask }}\right)$.

on scoring from +2 (hot) to -2 (cold), where neutral (0) ranges from a budget of -20 to $+150 \mathrm{~W} \mathrm{~m}^{-2}$. Figure 3 displays the frequency of scores, with a negative skew to the right (warm categories), which reflects the fact that 3 of the 4 test days were warm - hot, and subjects were preforming physical activity. Subjects ranked their ATS as neutral 57\% of the time, while the PTS was neutral 9.5\% and $17.3 \%$ using $\bar{T}_{A s k}$ and $\bar{T}_{P s k}$, respectively.

The strength of the relationship between ordinal ATS and PTS data using actual and predicted $T_{s k}$ was tested using a Spearman's rho correlation $\left(\mathrm{r}_{s}\right) . \mathrm{r}_{s}$ for the PTS votes using $T_{P s k}\left(\mathrm{r}_{s}=0.315\right)$ was slightly higher than that based on $T_{A s k}$ $\left(\mathrm{r}_{s}=0.285\right)$, both showing significance at the $\mathrm{p}<0.10$ level. This is opposite of what was expected since modelled $\bar{T}_{s k}$ measurements were less accurate; hence, using $\bar{T}_{P s k}$ values from the COMFA model should do a poorer job of estimating ATS, but it does a better job than $\bar{T}_{A s k}$. This suggests a problem elsewhere in the model, possibly due to differing physiological and psychological factors.

During the cycling tests, significant correlation of ATS with $\bar{T}_{P s k}$ was very low $\left(\mathrm{r}_{s}=0.243, \mathrm{p}<0.05\right)$ and not present for $\bar{T}_{A s k}$, whereas running displayed high correlation with both $\bar{T}_{A s k}$ and $\bar{T}_{P s k}\left(\mathrm{r}_{s}=0.562\right.$ and 0.481 , respectively, $\left.\mathrm{p}<0.05\right)$. These results show that running PTS votes were better predicted than cycling, even though running $\bar{T}_{P s k}$ were less accurate than cycling. The low correlation of ATS with both predicted and actual $\bar{T}_{s k}$ values used for thermal sensation scoring shows the inherent difficulty of predicting the TC of a human during exercise.

The meaning of 'comfort' changes while exercising due to differing factors, such as different hormones produced from the hypothalamus causing a sense of 
pleasure [14], increased bloodflow to the surface to increase convective loss [8], as well as individuals being prepared and expectant of uncomfortable conditions. Due to such physiological mechanisms, the ATS response by subjects when exercising in more likely to be in the neutral range. Such mentioned mechanisms are not considered in the COMFA model, as they are difficult to quantify, and depend on individual psychology.

The psychological variables impacting ATS when exercising outdoors include, but are not limited to, aesthetics, seasons, weather, expectations, socializing, perception, and readiness to exercise. Recent studies have attempted to associate psychological perception [21] with outdoor use; however, psychological aspects of exercising outdoors are limited in the literature. In order to integrate climatic variability, the adaptive predicted mean vote (aPMV) has been tested using adaptive coefficients [22]. This notion may be further applied to exercising, as an adaptive coefficient is needed to differentiate between low and high metabolically demanding activities outdoors, and can thus improve the agreement of ATS with PTS votes from both $\bar{T}_{A s k}$ and $\bar{T}_{P s k}$.

\section{Conclusion}

The prediction of environmental heat stress requires models to make use of human physiology and physical parameters of the environment to quantify human TC, as well as heat stress leading to heat strain and impending mortality. This study has compared accurate measurements of $\bar{T}_{s k}$ to those calculated by the COMFA outdoor model for users performing moderate-vigorous physical activity, resulting in low, but statistically significant, relationships between ATS and PTS scores. The COMFA model used in this study resulted in overestimation of actual $\bar{T}_{s k}$ measured from the subjects' skin; however, PTS using $\bar{T}_{P s k}$ had a slightly higher correlation with ATS scores than when using PTS with $T_{\text {Ask }}$.

Individuals accept broader thermal conditions when outdoors [3]. This study showed that with outdoor conditions plus exercise, individuals are even more tolerant of uncomfortable conditions, thus having broader comfort zones. The relationship between perceptive psychology and ATS during exercise is very difficult to quantify in human comfort models due to a variety of psychological and climatic factors, including expectations, adaptation, perceived control, fitness, body composition and size, age, gender, and time of year. The results of the present study support findings of past research with ATS and PTS due to these additional factors. Core temperature is a dominant physiological component of human thermoregulation, most importantly with sweat rate, secondarily with local $T_{s k}$ [23]; thus, $T_{c}$ prediction in TC models is also critical, particularly for heat stress.

Further research is required to integrate the knowledge of the climate-human behaviour relationship, and its implications for sustainable urban design. Heat related mortality statistics warrant increased use of climate-sensitive design in urban areas in order to avoid heat stress, prevent decreased work and exercise, as well as reduce detrimental impacts on sports performance [2]. It is important to underline that the 7-point TS model is subjective; hence, there is intra- and inter- 
personal variation within ATS votes. Psychological aspects in the outdoor climate have not been a large focus in human TC studies [21], and future research must clarify the variables associated with predicting the TC of humans while exercising outdoors.

\section{References}

[1] Brown, R.D. \& Gillespie, T.J., Estimating outdoor thermal comfort using a cylindrical radiation thermometer and an energy budget model. Int J Biometeorol, 30(1), pp. 43-52, 1986.

[2] Brotherhood, J.R., Heat stress and strain in exercise and sport. J Sci Med Sport, 11(1), pp. 6-19, 2008.

[3] Johansson, E. \& Rohinton, E., The influence of urban design on outdoor thermal comfort in the hot, humid city of Colombo, Sri Lanka. Int J Biometeorol, 51, pp. 119-133, 2006.

[4] Golden, J.S., Hartz, D., Brazel, A., Luber, G. \& Phelan, P., A biometeorology study of climate and heat-related morbidity in Phoenix from 2001 to 2006. International journal of biometeorology, 52(6), pp. 471-480, 2008.

[5] Gosling, S., McGregor, G. \& Lowe, J., Climate change and heat-related mortality in six cities part 2: Climate model evaluation and projected impacts from changes in the mean and variability of temperature with climate change. International journal of biometeorology, 53(1), pp. 31-51, 2009.

[6] Fanger, P.O., Thermal Comfort. Analysis and Application in Environmental Engineering. Danish Technical Press: Copenhagen, 1970.

[7] Gagge, A.P., An effective temperature scale based on a single model of human physiological temperature response, 1971.

[8] Huizenga, C., Hui, Z. \& Arens, E., A model of human physiology and comfort for assessing complex thermal environments. Build and Environ, 36(6), pp. 691-699, 2001.

[9] Hoppe, P., Different aspects of assessing indoor and outdoor thermal comfort. Energ Buildings, 34(6), pp. 661-665, 2002.

[10] Ramanathan, N.L., A new weighting system for mean surface temperature of the human body. J Appl Physiol, 19(3), pp. 531-533, 1964.

[11] Yao, Y., Lian, Z., Liu, W. \& Shen, Q., Experimental study on skin temperature and thermal comfort of the human body in a recumbent posture under uniform thermal environments. Indoor built environ, 16(6), p. 505, 2007.

[12] ISO9920, ISO 9920: Ergonomics of the Thermal Environment: Estimation of thermal insulation and water vapour resistance of a clothing ensemble. ISO: Geneva, 2007.

[13] Ainsworth, B.E., Haskell, W.L., Whitt, M.C., Irwin, M.L., Swartz, A.M., Strath, S.J. \& O'Brien, W.L., Compendium of Physical Activities: an update of activity codes and MET intensities. Med Sci Sport Exer, 32(9), pp. 498516, 2000.

[14] Havenith, G., Holmr, I. \& Parsons, K., Personal factors in thermal comfort 
assessment: clothing properties and metabolic heat production. Energ Buildings, 34(6), pp. 581-591, 2002.

[15] Borg, G.A.V., Psychophysical bases of perceived exertion. Medicine and Science in Sports and Exercise, 14, pp. 377-381, 1982.

[16] Kenny, N.A., Warland, J.S., Brown, R.D. \& Gillespie, T.J., Part A: Assessing the performance of the COMFA outdoor thermal comfort model on subjects performing physical activity. Int J Biometeorol, 415-428, pp. 415-428, 2009.

[17] Karvonen, M., Kentala, K. \& Mustala, O., The effects of training on heart rate: a longitudinal study. Ann Exp Biol Fenn, 35, pp. 307-267, 1957.

[18] Kenny, N.A., Warland, J.S., Brown, R.D. \& Gillespie, T.J., Part B: Revisions to the COMFA outdoor thermal comfort model for application to subjects performing physical activity. Int J Biometeorol, 53, pp. 429-441, 2009.

[19] Mairiaux, P., Malchaire, J. \& Candas, V., Prediction of mean skin temperature in warm environments. Eur J Appl Phys, 56(6), pp. 686-692, 1987.

[20] Sparks, S., Cable, N., Doran, D. \& Maclaren, D., The influence of environmental temperature on duathlon performance. Ergonomics, 48, pp. 1558 1567, 2005.

[21] Lin, T., Thermal perception, adaptation and attendance in a public square in hot and humid regions. Building and Environment, 44(10), pp. 2017-2026, 2009.

[22] Yao, R., Li, B. \& Liu, J., A theoretical adaptive model of thermal comfort Adaptive Predicted Mean Vote (aPMV). Building and Environment, 44(10), pp. 2089-2096, 2009.

[23] Shibasaki, M., Wilson, T.E. \& Crandall, C.G., Neural control and mechanisms of eccrine sweating during heat stress and exercise. J Appl Physiol, 100(5), pp. 1692-1701, 2006. 